\title{
Diabetic Retinopathycal Identification, Analysis and Diagnosis using Color Images
}

\author{
Vinitha K. \\ Research Scholar \\ Department of information technology \\ Bharathiar University, Coimbatore \\ Tamilnadu
}

\author{
M. Sundaresan, PhD \\ Professor and Head \\ Department of Information Technology \\ Bharathiar university, Coimbatore \\ Tamilnadu
}

\begin{abstract}
Image processing is deals with wave processing which the input data is a picture, and the result can be either an image or a set of parameters corresponding to the image. Medical Imaging is a wide technology which helps the doctors to see the interior portions of the body parts for easy diagnosis. The proposed system edge-based segmentation is applied for vessel extraction. It produces edge maps which are based on Kirsch edge detection methods. The proposed method discovers the eye disorder such as cardio vascular diseases and diabetic retinopathy. In order to evaluate the performance of proposed work, there are several statistical criteria which are related to the sensitivity, specificity, accuracy and precision.
\end{abstract}

\section{Keywords}

Biomedical Image Processing, Retinal Structure, vessel segmentation, diabetic retinopathy, Kirsch-Template, Retinal Disease Diagnosis.

\section{INTRODUCTION}

The use of image is done in most of the fields today consisting of image processing and computer vision. Almost all image processing techniques require serve the picture as two dimensional and standard signal-processing techniques used in to it. Medical imaging plays an important role in monitoring the patient and providing effective treatment. Biomedical image processing is a wide area; it extends over biomedical signal assembling, picture establishing, picture processing, and image display to medical diagnosis. Biomedical image processing has recently focused on forming computational tools for clinical decision support.

The kirsch Template is an effective method for medical diagnosis which is used here for vessel extraction and classification. To extract the blood vessels from retinal image is necessary for proper diagnosis. The human eye is a most important and complex organ. The retina is a layer of tissue in the back of eye that sense light and send images of the object to the brain. Digital retinal imaging is a new technology that can be used to access patients for diabetic retinopathy. [1].

\section{MATERIALS AND METHODS}

Segmentation techniques and methods are deviate, based on the imaging modality. There are some methods and techniques approachable to the genuine identification and extraction of vessels from retinal fundus images in first stages. The last phase of disease recognition may cause vision loss and blindness. The automated examination and screening procedure for detection of diabetic retinopathy are helpful for easy diagnosis. Retinal vessel segmentation is useful for diabetic retinopathycal diagnosis and treatments. [2].

\subsection{Identifying True Vessels from Segmented Images}

Qiangfeng peter Lau et al., "Simultaneously Identifying All True Vessels from Segmented Images" deals with the computation of retinal blood vessel structure to be related to the possibility of cardiovascular diseases. The wrong recognition of blood vessels may effect in a huge alternation of these computation, leading to a wrong clinical diagnosis. In this paper, the problem of automatically identifying true vessels act as a post processing step to vascular structure segmentation. Each vessel is followed separately by frequently finding the next vessel point with achieving function that examine the pixel potency and intension in the sector of the current point in the image.. [3].

\subsection{Automatic Approach for vessel classification}

BehdadDashtbozorg et al., "An Automatic Graph-Based Approach for Artery/Vein Classification in Retinal Images", deals with the classification of arteries and vein in retinal images. One major distinction is the reality that the technique is able to classify the whole vascular tree and does not regulate the classification to particular regions of interest, normally around the optic disc. The previous methods mostly use intensity feature for discerning between arteries and veins, current method use additional information extracted from graph which represents the vascular network. Comparing with other classifiers the k-Nearest Neibour (KNN) classifier provides the best overall performance. These methods mostly used for intensity features to differentiate between arteries and veins. [4].

\subsection{Separation of Arteries from Vein in Retinal Images}

Mirsharif et al., "Developing an Automatic Method for Separation of Arteries from Veins in Retinal Images", deals with extricate and review the best character from inner and outer segments of vessels for grouping of retinal blood vessels particularly the major ones with the allocation of blood vessels in RGB and HSL color spaces. Since most of consecutive points in a vessel segments have similar features and these points belong to same type of vessel we attempt to divide long vessel segments into smaller ones and extract features for each vessel segment instead of every centerline points. a group of disease such as heart stroke, hypertension, blood pressure have proven to show some signs in retinal blood vessels or make alternations to width of arteries and 
veins in different ways leading to abnormally thick arteries, thin veins or vice versa. [5].

\section{OBJECTIVES OF PROPOSED WORK}

The main attraction of image processing methods stems from two main application areas they are improvement of pictorial information for human interpretation; and processing of image data for storage, transmission and representation for autonomous machine perception. The main objective of the work is to introduce a computerized method which helps to extract the vein from retinal image to identify the nicking points and diabetic retinopathy from color fundus images.

\section{MEDICAL DIAGNOSIS USING KIRSCH TEMPLATE}

The kirsch template is used for recognizing the blood vessels from the retinal images. It is used to locate the edges and remove the noise from the fundus image. In the proposed work, the automated analysis and screening system for early detection diagnosis and treatment of diabetic retinopathy is very useful today.

Through computer simulations it is possible to demonstrate that prevention and treatments are relatively inexpensive compared to healthcare and rehabilitation costs incurred by vision loss or blindness. The affected parts from the image are separated for disease classification which forms the region of interest.

The edges are the boundaries between object and its background and the proposed algorithm, Kirsch template is used for segmentation of the vessels in all regulations and at different intention. Figure 1 shows the main steps of the proposed method. The kirsch template is mainly used in color images to filter the image and remove the noise from it.

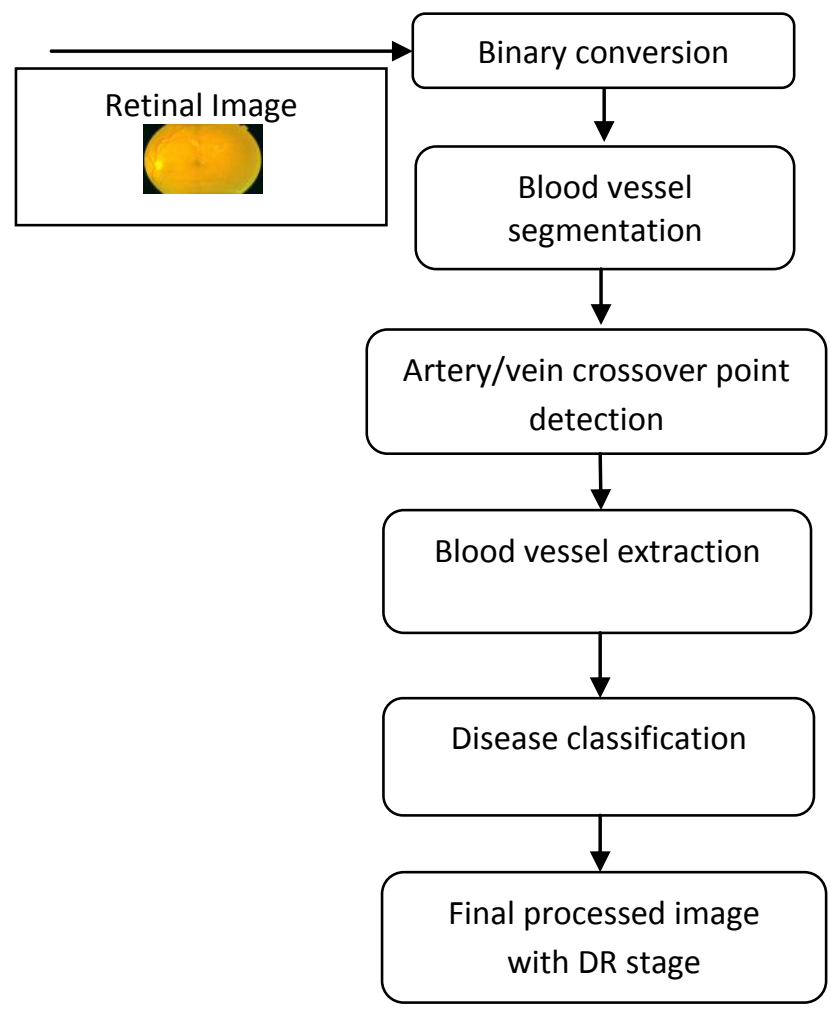

The sample retinal images are shown in figure 2 and 3 below. Proposed method performs as below:

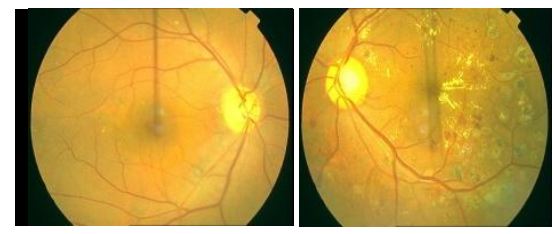

Fig 2 and Fig 3 are sample retinal images

Input image selection: The retinal image processing plays an important role in medical imaging. Retinal photographs are the main source for screening the diabetic retinopathy. Input image selection is the starting stage of diabetic retinopathy diagnosis.

Initial segmentation: After selecting an infected image from storage area, then moves to initial segmentation. It use edge based segmentation for extracting the vessels. It converted the color image into gray scale image and removes the noise from it. Then Initial segmentation and nicking identification consist of four major tasks, they are:

- Layer image: It has two functions; 1) major vessel identification, 2) venular segment identification.

The imported image has a lot of blood vessel layers. The color image is filtered and removes the noise and other unwanted background areas from it. The layer image shows that the blood vessels present in the image.

- Bandwidth image: The band width image shows that the thickness of each and every small veins and artery present in the fundus image and the difference in thickness and width is the identification of problem. If there are any swelling areas they are the symptoms of various diseases.

- Thinned image: The thinned image is used to find the minute veins and their crossovers are the next stage of the processing. Applying Neiborhood operation to find small veins and arteries present on the fundus image.

- Bifurcation image: The bifurcation is the last stage of initial segmentation to extract the infected veins and their crossover points separately with distance.

Hence, accurate extraction of vessels is becoming a main topic of this research area. After completing the initial segmentation task, the particular image is moved to next vein extraction task.

\section{Vessel Extraction}

The retina is a thin layer of tissue that contain large amount of different length blood vessels. Vein extraction is nothing but converting the color fundus image into gray scale image and subtracting the vessels from it .vein extraction is the process of separating the veins from fundus image.selecct the extraction of vein button from primary widow. Press on the extraction of vein button. The displayed widow has two images first one is the input image and next is its Skelton image. Blood vessel extraction is easy way to identifying small minute vessels from affected image and the extracted blood vessels of the input image.

Fig 1: Steps of the proposed work 


\section{Vessel Classification}

The proposed method evaluating the entire image and classifying the blood vessels based on vessel diameter. Image enhancement is used to improve the Image and dividing the veins based on its color. To classifying the vessels separately based on the vessel diameter the user can easily classify the small veins with using colors. The blood vessel classification is useful to find the same length vessels separately.

\section{Disease Classification}

Disease classification is the important step to analysis and diagnosis for effective treatments. Based on the ratio of vessels and estimated area of cotton wool spots identifying the stage of diabetic retinopathy. Analyze the retinal image and find diabetic retinopathy. Retinal disease diagnosis is an important task which is performed here with using proposed method. Disease classification is an important task to identify the seviority of diabetic retinopathy. The proposed method converting the selected image into its binary format and delete the unwanted areas from it for further processing.

During diabetic retinapathycal diagnosis the final resulting window is displayed with several aspects. They are:

Ratio of vessels: The amount of vessels present in the retinal image is called as ratio of vessels.

Estimated cotton wool spots: cotton wool spot is the sign of severe injuries presented in the nerve fibers in retina. Exudates related with patches of vascular damage with leakage. Disease classification is a main task in actual medical diagnosis.

Crossover points: The retinal image has so many crossing points and splits. To find the crossing points or joints are also useful for recognizing the measure of diabetic retinopathy.

Stage of eye: examination of diabetic retinapathycal diagnosis shows the present position of disease. The final result should be changed based on the input retinal image.

The retinal image processing plays an important role in medical imaging. The human retina having a lot of vessels, some are carrying deoxygenated dirty blood from body to the heart called vein and some are carrying oxygenated pure blood from heart to tissues in the body. The disease stage are evaluated Based on the result of ratio of cotton wool spots and ratio of damaged vessels. Based on the experimental result the physician can easily understand the current stage of disease and treat the patient properly.

\section{RESULT AND DISCUSSION}

Retinal image processing is an important tool for diagnosing and curing the diseases. The characteristic of vessels plays an important role in various medical diagnoses. To examine the retinal blood vessels can contribute very useful data to the doctors for early detection of diseases. The automated analysis and screening system for detection of diabetic retinopathy are helpful for diagnosis.

Diabetic-retinopathy diagnosis systems can replace manual methods and significantly reduce the manual labor involved in the screening process. The process of automatic diabetic retinopathy detection includes recognition and segmentation of the abnormal characteristic from the input images

\section{Performance evaluation}

The performance evaluation is an important task to evaluate the performance of proposed work. The proposed system processed by using 5 set of normal and abnormal images as input and showing the performance of new method based on certain parameter such as sensitivity, specificity, accuracy and precision. The Performance measuring data and graph with a normal and abnormal image are given below.

\section{Sensitivity:}

The sensitivity is used to evaluate a clinical experiment of statistics to narrate a diagnostic test. Sensitivity with normal image is shown in the table 1 and its sensitivity performance graph is shown in figure 4.The sensitivity using abnormal images is shown in figure 5 and its graph is shown in figure 6.

Sensitivity $=(\mathrm{TP} /(\mathrm{TP}+\mathrm{FN})) * 100$

TP - True positive (The patient has the disease and the test result is also true)

FN - False negative (The patient has the disease but the test result is negative)

Performance analysis with normal and abnormal images:

Table 1. Sensitivity table of the normal image

\begin{tabular}{|r|r|r|r|r|r|}
\hline & Image 1 & Image 2 & \multicolumn{1}{|c|}{ Image 3 } & Image 4 & Image 5 \\
\cline { 2 - 6 } Existing & 17.30 & 10.12 & 15.88 & 8.63 & 12.73 \\
\hline Proposed & 46.08 & 44.19 & 62.80 & 51.04 & 44.08 \\
\hline
\end{tabular}

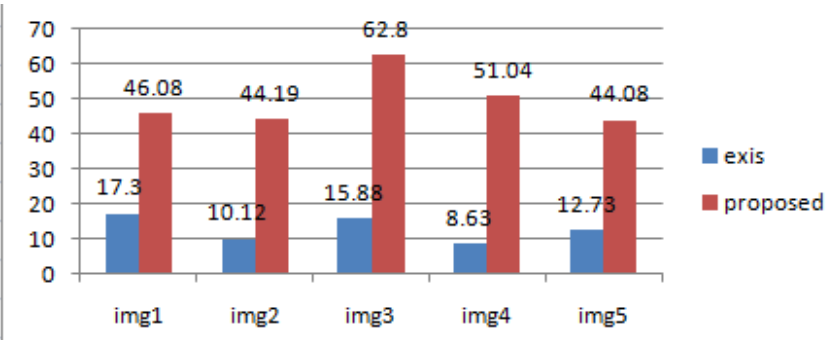

Fig 4: Sensitivity graph of the normal image

Table 2. Sensitivity table of the abnormal image

\begin{tabular}{|r|r|r|r|r|r|}
\hline & Image 1 & Image 2 & Image 3 & Image 4 & Image 5 \\
\cline { 2 - 6 } Existing & 19.13 & 16.88 & 13.54 & 12.88 & 16.28 \\
\hline Proposed & 32.29 & 40.12 & 41.35 & 16.56 & 41.86 \\
\hline
\end{tabular}

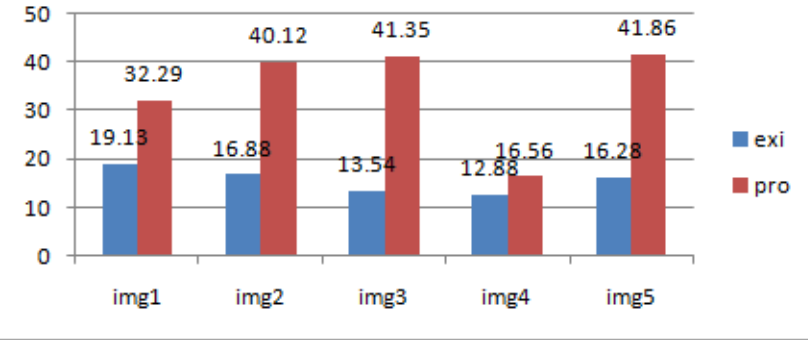

Fig 5: Sensitivity graph of the abnormal image

\section{Precision :}

The Precision describes the closeness of two or more measurements. It is also called as positive predictive value 
(PPV). The precision of the normal image is shown in the table 3 and its graph is shown in figure 6 . The precision result with abnormal image is shown in the table 4 and its graph is shown in figure 7.

Precision $\mathrm{PPV}=\mathrm{TP} /(\mathrm{TP}+\mathrm{FP})$

FP- False positive (The patient doesn't have the disease but the test result is positive)

Table 3. Precision table of the normal image

\begin{tabular}{|c|c|c|c|c|c|}
\hline \multirow{2}{*}{ Existing } & Image 1 & Image 2 & Image 3 & Image 4 & Image 5 \\
\cline { 2 - 6 } & 0.1474 & 0.1604 & 0.1225 & 0.1427 & 0.1474 \\
\hline Proposed & 0.7118 & 0.4391 & 0.9424 & 0.7158 & 0.7118 \\
\hline
\end{tabular}

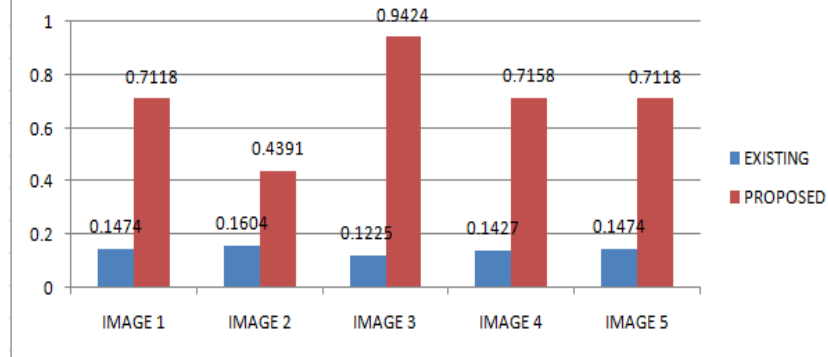

Fig 6: Precision graph of the normal image

Table 4. Precision table of the abnormal image

\begin{tabular}{|c|r|r|r|r|r|}
\hline \multirow{2}{*}{ Existing } & Image 1 & Image 2 & Image 3 & Image 4 & Image 5 \\
\cline { 2 - 6 } & 0.1975 & 0.1875 & 0.2267 & 0.1833 & 0.1989 \\
\hline Proposed & 0.2803 & 0.5986 & 0.5846 & 0.2956 & 0.5112 \\
\hline
\end{tabular}

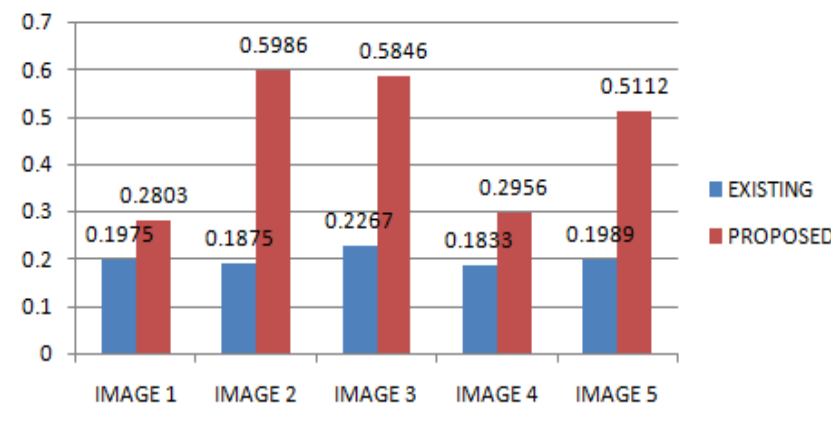

Fig 7: Precision graph of the abnormal image

\section{.Accuracy:}

The accuracy describes the quality of measurement is being correct . The accuracy result of the input normal image is shown in the table 5 and its graph is shown in figure 8 . The result of an abnormal image is shown in the table 6 and its graph is shown in figure 9 .

$$
\text { Accuracy }=((\mathrm{TP}+\mathrm{TN}) / \mathrm{P}+\mathrm{TN}+\mathrm{FP}+\mathrm{FN}))^{*} 100
$$

TN- True negative (The patient doesn't have the disease and the test result is also negative)
Table 5. Accuracy table of the normal image

\begin{tabular}{|c|c|c|c|c|c|}
\hline \multirow{2}{*}{ Existing } & $\begin{array}{c}\text { Image } \\
1\end{array}$ & $\begin{array}{c}\text { Image } \\
2\end{array}$ & $\begin{array}{c}\text { Image } \\
3\end{array}$ & $\begin{array}{c}\text { Image } \\
4\end{array}$ & $\begin{array}{c}\text { Image } \\
5\end{array}$ \\
\cline { 2 - 6 } & 88.19 & 86.43 & 89.33 & 87.76 & 82.19 \\
\hline Proposed & 92.89 & 95.06 & 96.76 & 98.99 & 96.89 \\
\hline
\end{tabular}

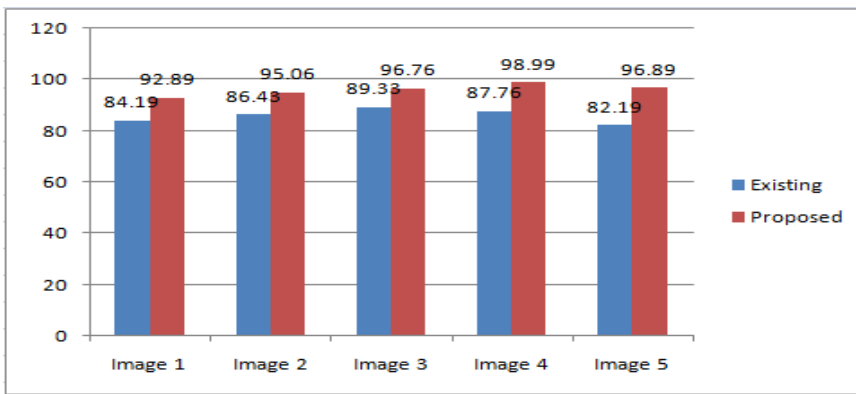

Fig 8: Accuracy graph of the normal image

Table 6. Accuracy table of the abnormal image

\begin{tabular}{|c|c|c|c|c|c|}
\hline \multirow{2}{*}{ Existing } & $\begin{array}{c}\text { Image } \\
1\end{array}$ & $\begin{array}{c}\text { Image } \\
2\end{array}$ & $\begin{array}{c}\text { Image } \\
3\end{array}$ & $\begin{array}{c}\text { Image } \\
4\end{array}$ & $\begin{array}{c}\text { Image } \\
5\end{array}$ \\
\cline { 2 - 6 } & 86.80 & 88.13 & 80.18 & 81.58 & 83.97 \\
\hline Proposed & 91.70 & 90.29 & 93.12 & 88.80 & 87.13 \\
\hline
\end{tabular}

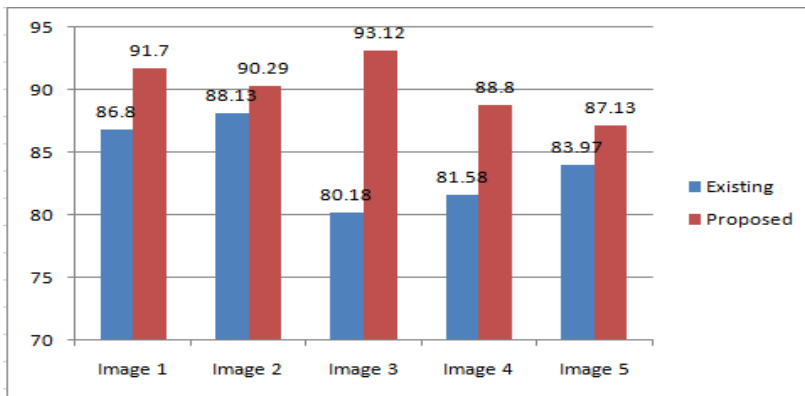

Fig 9: Accuracy graph of the abnormal image

\section{CONCLUSION}

Retinal disease diagnosis is an important task which is performed here with using proposed method. MATLAB is a user friendly computing techniques that yield effortless programming for user and produce higher quality experimental result in medical image processing. The proposed method converts the color image into its binary format. Then kirsch's template with spatial filtering is used to find the orientation and direction of the blood vessels and boundaries. The proposed system applied edge-based segmentation for vessel extraction and disease identification. It produces edge maps which are based on Kirsch edge detection method. Finally the Kirsch template produces better experimental results than existing method.

\section{FUTURE ENHANCEMENT}

In order to analyze the performance of new work, the proposed system produce better accuracy, sensitivity and precision than the existing method. In future, the research 
can be continued and produces better performance, measurements and classifications using Neural Networkbased approaches.

\section{REFERENCES}

[1] Rafael C Gonzalez and Richard E Woods (2013), “Digital Image Processing”, ISBN 978-81-317-2695-2, Pearson education, pp. 1-24 and 36-37.

[2] Mohd.imran khan, heena sheikh, Anwar mohd.mansuri, pradhumn soni, "a review of retinal vessel segmentation techniques and algorithms", international journal of computer technology and application, volume 2(5), september-october 2011, pp. 1
[3] Qiangfeng peter Lau, mong li lee, wynne hsu*, and Tien yin Wong, "simultaneously identifying all true vessels from segmented retinal images", IEEE Transactions on biomedical engineering, 2013, pp. 1-6.

[4] Behdad Dashtbozorg, Ana Maria Mendonça, "an automatic graph-based approach for artery/vein classification in retinal images", IEEE transactions on image processing, volume 23, no.3, March 2014, pp. 110.

[5] G.Mirsharif, F.Tajeripour, Sobhanmanesh, H.Pourreza, T.Banaee, "developing an automatic method for separation of arteries from veins in retinal images", international conference on computer and knowledge engineering (ICCKE), october13-14, 2011, pp.1-15. 